\title{
Measuring Developer Contribution from Software Repository Data
}

\author{
Georgios Gousios Eirini Kalliamvakou Diomidis Spinellis \\ Department of Management Science and Technology \\ Athens University of Economics and Business \\ \{gousiosg,ikaliam,dds\}@aueb.gr
}

\begin{abstract}
Apart from source code, software infrastructures supporting agile and distributed software projects contain traces of developer activity that does not directly affect the product itself but is important for the development process. We propose a model that, by combining traditional contribution metrics with data mined from software repositories, can deliver accurate developer contribution measurements. The model creates clusters of similar projects to extract weights that are then applied to the actions a developer performed on project assets to extract a combined measurement of the developer's contribution. We are currently implementing the model in the context of a software quality monitoring system while we are also validating its components by means of questionnaires.
\end{abstract}

\section{Categories and Subject Descriptors}

D.2.8 [Software Engineering]: Metrics-Process metrics; D.2.9 [Software Engineering]: Management-Productivity

\section{General Terms}

Measurement, Management

\section{Keywords}

Contribution, Software repositories

\section{INTRODUCTION}

An important aspect of all engineering principles is the assessment of the contribution of individuals that work on a project. Contribution assessments are performed to monitor the rate of project development, identify implementation bottlenecks and isolate exceptional cases, while the results of contribution assessments can help with future project planning. A common problem with contribution assessment is the definition of what contribution is in a particular context

Permission to make digital or hard copies of all or part of this work for personal or classroom use is granted without fee provided that copies are not made or distributed for profit or commercial advantage and that copies bear this notice and the full citation on the first page. To copy otherwise, to republish, to post on servers or to redistribute to lists, requires prior specific permission and/or a fee.

MSR'08, May 10-11, 2008, Leipzig, Germany.

Copyright 2008 ACM 978-1-60558-024-1/08/05 ...\$5.00. and also the selection and application of the appropriate measurements.

In software engineering, contribution assessment entails the measurement of the contribution of a person in terms of lines of code or function points towards the development of a software project [7]. In the recent years, the shift to more agile development practices and the proliferation of software and project management tools has reduced the estimation capacity of classic software estimation models. A software developer today is not only required to write code, but also to communicate with colleagues effectively and to use a variety of tools that produce and modify code with minimal input from his side. This change has become more apparent with the emergence of Open Source Software (oss).

In this paper, we propose a new model for evaluating developer contribution, which takes advantage of software development repositories to extract contribution indicators.

\section{EXISTING WORK}

Despite how central the notion of contribution is to software development (especially oss) it is not a central theme in related literature in itself. Developer contribution is discussed in several contexts and is often related to other notions such as productivity, participation or activity.

Productivity in abstract terms is the rate of output per unit of input; it is essentially a measure of production efficiency. Productivity evaluation usually entails extrapolating a measurable quantity of a unit of work over the resources required to produce it. In software engineering, productivity has been a long-studied issue, as it has both managerial and engineering aspects.

An important, and still open, issue is the definition of what a unit of work is in the context of software engineering. In one of the first studies appearing in the literature [11], Sackman refers to words as the unit of work. The programming code is viewed as literature text and each spaceseparated identifier is considered to be a unit of thought. As engineers started to understand that programming language constructs cannot be classified as intellectual work, the unit of work definition shifted to lines of code (LOC), which still remains the most widely used measure of software size. A weakness of the LOC metric is that it can only be determined safely at the end of a project. To that end, function points analysis usually complements the LOC metric as a unit of work for software estimation. Other units of work that have been proposed in the literature are classes, methods and components. A discussion on the various units of work in the context of object oriented software can be found in [3]. 
There is no clear definition of what is contribution in the context of software development. The project resources that usually receive input are identified in references [6] and [9]. Software developers participate mainly by writing code, but also by following discussions and submitting comments and ideas to mailing lists as well as performing bug fixes [10, 13]. As a result, the source code repository, the mailing list archives and bug databases are the widely used data sources for discussing participation and activity in a software project.

In reference [4], Glass et al. propose a distinction between clerical and intellectual types of actions in software development. Drawing on contemporary literature, the paper reports extended taxonomies of activities, spanning the software development process from its initial stages to its later ones. Our proposed asset/action taxonomy is in agreement with their lists of software tasks.

Finally, Amor et al. present a model for development effort estimation based on data extracted from software process traces [1]. Their model bears some similarity with our model in the basic idea of using repositories to extract process data, but their focus is on estimation rather than effort and contribution evaluation.

\section{OUR APPROACH}

Our work is concerned with the measurement of developer involvement and activity in the face of agile and distributed development practices. The model we are building exploits the availability of publicly accessible software repositories to perform measurements and identify possible trends by combining data across projects. It can also run fully automatically with no human intervention.

Our model departs from the classic measurement practices as it does not consider source code as the only contribution metric. This is a deliberate choice that we believe reflects better on how software is developed in an agile context, where an important portion of development time is spent on communication and manipulation of development support tools. Our model does not completely neglect the importance of source code either; we still use the lines of

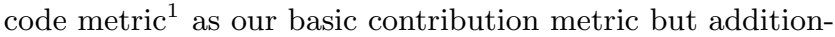
ally we also scale it according to a factor that results from the combination of the developer's actions on project assets. For a developer $d$, the contribution function $C(d)$ is defined as the sum of the total lines of code for the developer $L o C(d)$ plus the contribution factor function $C F(d)$.

$$
C(d)=\operatorname{LoC}(d)+C F(d)
$$

The important part of our model is the definition of the contribution factor function. To identify which actions can be classified as contribution, we follow a hierarchical, topdown approach: we first identify the project assets that can potentially receive contribution and then analyze the actions that can be performed on each of the identified assets to see if they constitute a contribution or not. The actions are initially identified intuitively and through personal experience; in Section 5, we present the method we will use for validating our selection of actions. Also, not all actions have a positive effect on a project; for example, a commit to a source code

\footnotetext{
${ }^{1}$ By "lines of code" we refer to non-comment program statements.
}

repository that leads to a bug or decreases the quality of the project as it is measured by metrics might be considered a negative contribution. Furthermore, not all actions have the same importance on the evolution of a project; for this reason, we also specify weights that are attached to each action.

In Table 1, we present a non-exhaustive breakdown of actions that can be performed on the identified project assets. Most actions are self-explanatory and relatively easy to mine from each asset repository using simple heuristics or external tools [12]. Each action is a measurable entity whose value is updated after the corresponding project asset has been updated. Values are calculated per developer and are aggregated per project asset. As each project asset might have a different importance for each project, we also assign a weight to the contribution of each asset to the total score for a project, as seen in equation 2 . The value $A^{x}$ denotes the sum of all the events affecting asset $x$ in the project's lifetime.

$$
A_{\text {total }}=\alpha * A^{r e p}+\beta * A^{m l}+\gamma * A^{b u g}+\delta * A^{w i k i}+\epsilon * A^{i r c}
$$

The values of the weights $\alpha, \beta, \gamma \delta$ and $\epsilon$ are calculated as the percentage of the sum of events for an asset in the total number of events, across projects. Individual projects are also allowed to assign project-specific values to the weights to account for what each project deems important for its development process. In the latter case, the value of $A_{\text {total }}$ is not of any particular use. As an interesting side effect however, in the former case, the $A_{\text {total }}$ metric gives us an estimate of the overall project activity, which we can then use to rank projects according to.

Each asset type sum is decomposed to an aggregation of measurable entities weighted individually. As an example, equation 3 presents an expanded version of the $A_{\text {rep }}$ aggregation. We denote $A_{i}$ the total number of actions for action $i$. We also assign a weight $w_{i}$ to each action type. Depending on whether an action has a negative or a positive contribution to the project, its value is added or retracted from the total score. Action aggregates for the remainder of the assets are expanded similarly.

$$
A^{r e p}=\sum_{i=0}^{n} w_{i} A_{i}=w_{1} C A L \pm w_{2} C N S \pm \ldots \pm w_{11} C B N
$$

The most complicated aspect of our model is the calculation of the weights $\left(w_{i}\right)$ for each action. For that, we use an approximative approach, exploiting the fact that the system this model is built for will extract information from many projects. Based on findings by Capiluppi et al. [2], we expect projects belonging to a single application domain to exhibit similar action patterns. For example, code development tools projects usually receive less documentation commits and feature more active mailing lists than educational projects. For this reason, we first create clusters of similar projects and then for each application cluster, we extract the action weights using the following algorithm:

1. Count all events for each actionID across all cluster projects

2. Count all events for all cluster projects 


\begin{tabular}{|c|c|c|c|}
\hline Asset & Action & ID & Type \\
\hline Code and & Add lines of code of good/bad quality & CAL & $\mathrm{P} / \mathrm{N}$ \\
\hline Documentation & Commit new source file or directory & CNS & $\mathrm{P}$ \\
\hline \multirow[t]{10}{*}{ Repository } & Commit code that generates/closes a bug & $\mathrm{CCB}$ & $\mathrm{N} / \mathrm{P}$ \\
\hline & Add/Change code documentation & $\mathrm{CAD}$ & $\mathrm{P}$ \\
\hline & Commit fixes to code style & $\mathrm{CSF}$ & $\mathrm{P}$ \\
\hline & Commit more than $X$ files in a single commit & $\mathrm{CMF}$ & $\mathrm{N}$ \\
\hline & Commit documentation files & $\mathrm{CDF}$ & $\mathrm{P}$ \\
\hline & Commit translation files & $\mathrm{CDF}$ & $\mathrm{P}$ \\
\hline & Commit binary files & $\mathrm{CBF}$ & $\mathrm{N}$ \\
\hline & Commit with empty commit comment & $\mathrm{CEC}$ & $\mathrm{N}$ \\
\hline & Commit comment that awards a pointy hat & $\mathrm{CPH}$ & $\mathrm{P}$ \\
\hline & Commit comment that includes a bug report num & $\mathrm{CBN}$ & $\mathrm{P}$ \\
\hline Mailing lists - & First reply to thread & MRT & $\mathrm{P}$ \\
\hline \multirow[t]{3}{*}{ Forums } & Start a new thread & MST & $\mathrm{P}$ \\
\hline & Participate in a flamewar & MFW & $\mathrm{N}$ \\
\hline & Close a lingering thread & $\mathrm{MCT}$ & $\mathrm{P}$ \\
\hline \multirow[t]{4}{*}{ Bug Database } & Close a bug & BCL & $\mathrm{P}$ \\
\hline & Report a confirmed/invalid bug & BRP & $\mathrm{P} / \mathrm{N}$ \\
\hline & Close a bug that is then reopened & BCR & $\mathrm{N}$ \\
\hline & Comment on a bug report & $\mathrm{BCR}$ & $\mathrm{P}$ \\
\hline \multirow[t]{3}{*}{ Wiki } & Start a new wiki page & WSP & $\mathrm{P}$ \\
\hline & Update a wiki page & WUP & $\mathrm{P}$ \\
\hline & Link a wiki page from documentation/mail file & WLP & $\mathrm{P}$ \\
\hline \multirow[t]{2}{*}{ IRC } & Frequent participation to IRC & IFP & $\mathrm{P}$ \\
\hline & Prompt replies to directed questions & IRQ & $\mathrm{P}$ \\
\hline
\end{tabular}

Table 1: Project resources and actions that can be performed on them. The Type column denotes whether an action has positive $(P)$ or negative $(N)$ impact.

3. The percentage of contribution of each action category to the total number of actions is the weight $w_{i}$ for each action

We expect large projects to have thousands of events in each action type and therefore the values that we will be able to obtain through our heuristic method will be close to those that we would have obtained from a linear regression analysis of those weights, should that have been possible. An evaluation of a large number of projects for each cluster will provide us with indicative information about which actions are most prominent in software development and will allow us to filter out those actions that do not have a significant effect on developer contribution.

Finally, the contribution factor $C F(d)$ is a specialization of equations 2 and 3 per developer. To calculate the contribution factor value, we count the number of events for each event type and for each asset type that were performed by a specific developed $d$. If $A_{i}^{a}(d)$ is a function that returns the number of actions of type $i$ performed by developer $d$ on asset $a$ and $w_{i}^{a}$ is the weight of action $i$ on asset $a$, then the contribution factor for the developer is calculated as show in equation 4.

$$
C F(d)=\alpha \sum_{i=0}^{n} w_{i}^{r e p} A_{i}^{r e p}(d)+\cdots+\epsilon \sum_{i=0}^{n} w_{i}^{i r c} A_{i}^{i r c}(d)
$$

\section{IMPLEMENTATION}

The model presented is being developed as an extension of the SQO-OSS system [5], and more specifically, as a plug-in to the Alitheia software evaluation tool. The Alitheia platform is an OsGi-based tool, targeted to the evaluation of software quality. It consists of a set of core services, such as accessors to project assets, continuous updating of monitored projects and relational data storage, and it is extensible through the use of plug-ins. Plug-ins can either implement basic software metrics or combine the results from other plug-ins arbitrarily. In fact, the contribution metric implementation is a compound plug-in, building on existing plug-ins that calculate basic metrics such as lines of code and mailing list participation. The system is designed to perform in-depth analysis of thousands of projects on a per repository revision basis and allows full automation of the quality evaluation process after the initial project registration.

\section{EVALUATION AND CALIBRATION}

To evaluate the validity of the proposed metric, we applied the Kaner and Bond metric evaluation framework [8]. The results can be seen in Table 2. The proposed metric captures well the scaling of the measured attribute. A careful selection of the metric components, i.e. the actions to be measured, will strengthen the metric's ability to produce accurate measurements.

To this end, we will combine questionnaires with statistical analysis. Questionnaires will solicit the views of developers (seen also as experts) regarding project assets and actions that provide contributions. Developers will be asked:

- to scale the importance of each action for a project

- to answer how these actions can be categorized into types

- to scale what the actions' perceived compatibility is with the given project assets

As a side-effect, respondents will also shed light on missing or redundant actions. Finally, we will use correlation analysis to identify and filter out actions that exhibit strong correlation coefficient. 


\begin{tabular}{|c|c|}
\hline Criterion & Our Metric \\
\hline Purpose & Assess developer contribution in agile and distributed working environments. \\
\hline Scope & A project developed by a distributed workgroup \\
\hline Measured Attribute & Degree of contribution to the development process \\
\hline Attribute Scale & Ratio scale \\
\hline Attribute Variability & $\begin{array}{l}\text { There is no knowledge of the variability of the measured attribute prior to perform- } \\
\text { ing the measurements }\end{array}$ \\
\hline Metric Function & $\begin{array}{l}\text { The proposed metric counts and weights the number of actions on project assets } \\
\text { and the lines of code on a per developer basis. The highest those counts are, the } \\
\text { more a developer has contributed to a project (see section } 3 \text { ) }\end{array}$ \\
\hline Metric Scale & $\begin{array}{l}\text { Ratio scale: The higher the contribution value, the more a developer has offered to } \\
\text { the project. }\end{array}$ \\
\hline Variability of readings & $\begin{array}{l}\text { Some metric components are based on heuristics which may not work in certain } \\
\text { cases. This may affect measurements in non-foreseeable ways. Metric components } \\
\text { showing unstable results should be identified and excluded from the final version of } \\
\text { the model. }\end{array}$ \\
\hline $\begin{array}{l}\text { Attribute and Metric } \\
\text { Relationship }\end{array}$ & $\begin{array}{l}\text { The metric generally captures changes in the attribute well. Metric components } \\
\text { are analogous to contribution, subject to variability. For } 2 \text { given developers } d 1 \text { and } \\
d 2 \text {, the equation } c(d 1)+c(d 2)=c(d 1+d 2) \text { is always valid. }\end{array}$ \\
\hline Side effects & $\begin{array}{l}\text { No side effects can be foreseen. As the metric takes into account a variety of factors } \\
\text { and it is automatically calculated it is difficult for developers to change their be- } \\
\text { havior towards optimizing the metric without increasing their actual contribution. }\end{array}$ \\
\hline
\end{tabular}

Table 2: Metric evaluation according to the Kaner and Bond framework

\section{CONCLUSIONS}

We presented a approach for evaluating developer contribution to the software development process, based on data acquired from software repositories and collaboration infrastructures. Our model introduces a set of predefined actions and exploits the availability of a large number of evaluated projects in the Alitheia tool to extract a weight of the importance of each action. As of this writing, we are implementing a plugin for a quality evaluation tool that produces measurements for each predefined action, while also recalculating the weights on regular intervals.

\section{ACKNOWLEDGEMENTS}

The authors would like to thank Ioannis Samoladas for his help and constructive comments. This work is supported by the European Community's Sixth Framework Programme under the contract IST-2005-033331 "Software Quality Observatory for Open Source Software" (SQO-OSS).

\section{REFERENCES}

[1] J. J. Amor, G. Robles, and J. M. Gonzalez-Barahona. Effort estimation by characterizing developer activity. In The 8th international workshop on economics-driven software engineering research. ACM, May 2006.

[2] A. Capiluppi, P. Lago, and M. Morisio. Characteristics of open source projects. In Proceedings of the Seventh European Conference on Software Maintenance and Reengineering, pages 317-327, Mar 2003.

[3] D. N. Card and B. Scalzo. Measurement for object-orienated software projects. In Proceedings of the 6th International Symposium on Software Metrics, Florida, Nov 1999.

[4] R. L. Glass, I. Vessey, and S. A. Conger. Software tasks: intellectual or clerical? Inf. Manage., 23(4):183-191, 1992.

[5] G. Gousios, V. Karakoidas, K. Stroggylos, P. Louridas, V. Vlachos, and D. Spinellis. Software quality assesment of open source software. In
Proceedings of the 11th Panhellenic Conference on Informatics, May 2007.

[6] G. Hertel, S. Niedner, and S. Herrmann. Motivation of software developers in open source projects: an internet-based survey of contributors to the linux kernel. Research Policy, 32(7):1159-1177, Jul 2003.

[7] S. H. Kan. Metrics and Models in Software Quality Engineering, chapter 12.3 Productivity Metrics. Addison-Wesley, 2003.

[8] C. Kaner and W. Bond. Software engineering metrics: What do they measure and how do we know? In 10th International Software Metrics Symposium (METRICS 2004). IEEE, IEEE CS Press, Sep 2004.

[9] S. Koch and G. Schneider. Results from software engineering research into open source development projects using public data. Diskussionspapiere zum tätigkeitsfeld informationsverarbeitung und informationswirtschaft, Wirtschaftsuniversität Wien, 2000.

[10] A. Mockus, R. T. Fielding, and J. Herbsleb. A case study of open source software development: the apache server. In ICSE '00: Proceedings of the 22nd international conference on Software engineering, pages 263-272, New York, NY, USA, 2000. ACM.

[11] H. Sackman, W. J. Erikson, and E. E. Grant. Exploratory experimental studies comparing online and offline programming performance. Commun. $A C M, 11(1): 3-11,1968$.

[12] D. Spinellis. Global software development in the FreeBSD project. In P. Kruchten, Y. Hsieh, E. MacGregor, D. Moitra, and W. Strigel, editors, International Workshop on Global Software Development for the Practitioner, pages 73-79. ACM Press, May 2006.

[13] G. von Krogh, S. Spaeth, and K. R. Lakhani. Community, joining, and specialization in open source software innovation: a case study. Research Policy, 32(7):1217-1241, Jul 2003. 